von Hitze, Luft und Licht gearbeitet. Mit anderen Worten, ich habe mir als Aufgabe gestellt, klarzulegen, welche Körper im Theer vorhanden siad, und nicht, welche Körper man aus dem Theer erhalten kann, welche beiden Fragen sich der Unbeständigkeit der Theerbestandtheile wegen nicht mit einander decken.

Was die Zusammenstellung anbetrifft, kann hier und da das Aufgezeichnete als überflüssig aufgefasst werden, weil es für die Resultate keine directe Anwendung gefunden hat. Ich hoffe aber, durch diese Angaben Anhaltspunkte für die weitere Forschung gegeben zu haben, abgesehen davon, dass ich selbst die Absicht hege, die Untersuchungen über Holztheer weiter zu verfolgen.

Früher glaubte man, dass bei der trocknen Destillation von Holz sich erst Acetylen bilde und später bei den böheren Temperaturen durch Polymerisation die grösseren Atomcomplexe. Dieser Auffassung wird in letzter Zeit widersprochen, wenigstens scheint es sicher zu sein, dass die aromatischen Verbindungen direct gebildet werden. Wie das sich nun auch verhalten mag, interessant war es, bei den vielen Manipulationen der vorliegenden Arbeit, zu beobachten, welche Veränderungen die Temperatur in den im Theer vorhandenen Körpern hervorrief. Sicher müsste man viele Körper, die jetzt fast nutzlos sind, durch Behandlung mit höheren (bezw. niedrigeren) Temperaturen, - z. B. Leiten durch Röhren, deren Temperatur reguliert wird -in nützliche verwandeln können.

Wie man allmählich über die in Holztheeren enthaltenen Verbindungen Uebersicht bekommt, und über die Entstehung der chemischen Producte bei der Theerbildung zur Klarheit gelangt, so wird man es auch mit der Zeit mehr und mehr in seine Gewalt bekommen, die erwünschten Körper in grösserer Ausbeute zu erhalten.

\section{Der Anapait, ein neues Kalkeisenphosphat von Anapa am Schwarzen Meere.}

Von Dr. A. Sachs, Assistent am mineral. Univ.-Inst. zu Breslau.

Herr Dr. Krantz in Bonn erhielt durch einen Privatsammler ein Mineral mit folgendem Bericht: "Ich sende Ihnen zwei Exemplare eines von mir auf einer Reise nach Ciskaukasien gefundenen Minerales, sowie einen Steinkern von Limonit, der mit feinen Krystallen desselben Minerals bezogen ist. Dieses Mineral scheint nach meiner vorläufigen (oberflächlichen) Analyse bisher unbekannt gewesen zu sein, denn ich finde in keinem meiner
Handbücher eine derartig krystallirende Combination von Kalk + Eisenoxydulphosphat. l)er Fundort ist ein oolithisches Limonitlager bei Anapa aun Schwarzen Meer." Und in einem späteren Briefe theilt derselbe Herr mit, dass der Kalkgehalt seiner Schätzung nach mindestens 25 Proc. betrage, dass ausser Phosphorsäure noch eine andere (vielleicht organische Säure) in kleiner Menge in dem Mineral vorhanden zu sein scheine, dass das auggetriebene und überdestillirte Krystallwasser stark sauer reagire, und dass die Exemplare des neuen Minerals die Fisengrube Scheljesni Rog, zwischen Taman und Anapa gelegen, zum Fundort hätten.

Herr Prof. Dr. Hintze bestätigte durch Autopsie die Neuheit des Minerals, dessen nähere Untersuchung er mir freundlichst überliess.

Bei qualitativer Analyse wurde entsprechend den Angaben des Sammlers Kalk, Eisen, Phosphorsäure und stark sauer reagirendes Krystallwasser gefunden, nur von einer organischen Säure - die ja auch nur in Spuren vorhanden sein sollte - war nichts zu bemerken. Woher das Krystallwasser sauer reagirte, war nicht zu ermitteln, vielleicht war es etwas $\mathrm{P}_{2} \mathrm{O}_{5}$. Die quantitative Analye des in Wasser unlöslichen, in kalten Säuren aber leicht löslichen Minerales ergab folgende Zahlen:

$$
\begin{aligned}
& \mathrm{Fe}=18,07 \text { Proc. } \\
& \mathrm{CaO}=27,77- \\
& \mathrm{P}_{2} \mathrm{O}_{5}=35,51- \\
& \mathrm{H}_{2} \mathrm{O}=18,47- \\
& \text { Spur von Alkalien } \\
& \text { Summa: } 99,82 \text { Proc. }
\end{aligned}
$$

$\mathrm{Da}$ in dieser Analyse die Basen genau in dem Verhältniss zur Säure stehen, wie es der Formel $\mathrm{R}_{3} \mathrm{PO}_{4}$ entspricht, so handelt es sich hier um ein normales Salz der Orthophosphorsäure, und der gesammte Wassergehalt ist als $\mathrm{K}_{r y s t a l l w a s s e r}$ anzusehen, was auch noch dadurch bestätigt wird, dass alles Wasser bei etwas über $120^{\circ}$ entweicht. Daraus ergiebt sich, dass das Mineral mit 4 Mol. Wasser krystallisirt, und dass ihm die Formel $\mathrm{Fe} \mathrm{Ca}_{2}\left(\mathrm{PO}_{4}\right)_{3}+4 \mathrm{H}_{2} \mathrm{O}$ zuzuschreiben ist, der theoretisch folgende Werthe entsprechen:

$$
\begin{gathered}
\mathrm{Fe} O=18,09 \text { Proc. } \\
\mathrm{Ca} \mathrm{O}=28,14- \\
\mathrm{P}_{2} \mathrm{O}_{5}=3 \overline{5}, 68- \\
\mathrm{H}_{3} \mathrm{O}=18,09- \\
\text { Summa: } 100,00 \text { Proc. }
\end{gathered}
$$

Härte zwischen 3 und 4.

Spec. Gewicht $=2,81$.

Vor dem Löthrohr auf Kohle rostfarbener Beschlag von Eisenoxyd bemerkbar, charakteristische Flammenfärbung nicht zu beobachten. 
Sucht man in Groth's tabellar. Übersicht der Mineralien (1. Aufl. 1898) nach schon bekannten wasserhaltigen normalen Phosphaten, die einige Ähnlichkeit mit der chemischen Zusammensetzung des neuen Minerales aufweisen, so kommen nur zwei in $\mathrm{Be}-$ tracht: das ist der triklin krystallisirende Fairfieldit $=\left(\mathrm{PO}_{4}\right)_{2}(\mathrm{Ca}, \mathrm{Mn}, \mathrm{l} \cdot \mathrm{e})_{3}+2 \mathrm{H}_{2} \mathrm{O}$, der jedoch nach den Analysen von Penfield und Wells etwa 17 Proc. Mn $O$ enthält und deswegen ausscheidet, und der von Muthmann und Spiegel untersuchte Messelit, dem die Formel $\left(\mathrm{PO}_{4}\right)_{2}(\mathrm{Ca}, \mathrm{Fe}, \mathrm{Mg})_{3}+2^{1 / 2} \mathrm{H}_{2} \mathrm{O}$ zukommt, der also sowohl chemisch, wie übrigens auch krystallographisch, vom Anapait abweicht, wenn auch gewisse krystallographische Äbnlichkeiten zu constatiren sind.
Bezüglich der geologisch-mineralogischen Verhältnisse sei bemerkt. dass der vorher erwähnte Steinkern von Limonit (der mit Anapait überzogen ist) von Herrn Dr. Wys ogórski als dem Tertiär angehörig und zwar als Cardium (cf. acardo Desh.) bestimunt wurde. Das Krystallsystem des Anapaits ist das trikline, die Krystalle sind dünntafelig nach einer zur Qucrfläche gewählten Fläche ausgebildet. Auch das optische Verhalten deutet $z$ weifellos auf das trikline System. Bezüglich der näheren krystallographischen und optischen Bestimmungen mag hier nur auf meine Mittheilungen in den am 16. Januar 1902 herausgegebenen Sitzungsberichten der Königlich Preussischen Akademie der Wissenschaften zu Berlin verwiesen werden.

\section{Sitzungsberichte.}

Sitzung der Chemical Society. Vom 16. Januar 1902.

Vorsitzender: Prof. Emerson Reynolds. E. Rutherford und F. Soddy lesen über die radioactive Ausstrahlug von Thorinmverbindungen. Die zur Messung der Ausstrahlung angewandte Methode war eine elektrische und gestattete sowobl quantitative als auch qualitative Schlüsse. Die Vortragenden stellten sich die folgenden 3 Fragen zur Untersuchung: 1. Kann Thorerde, deren Ausstrahlungsfähigkeit durch Glühen zum grössten Theil zerstōrt worden ist, diese Eigenschaft durch chemische Behandlung wieder erhalten? 2. Ist die Ausstrablungsfähigkeit eine specifische Eigenschaft des Thoriums oder ist dieselbo der Gegenwart einer anderen Substanz zuzaschreiben? 3. Ist die radioactive Ausstrahlung in ihrem chemischen Verbalten einer bekannten Substanz ãhnlich? - Die erste Frage konnte bejahend beantwortut werden. In Bezug auf die zweite Frage war es den Vortragenden nicht möglich, eine Substanz ans der Thorerde abzuscheiden, welcher das Ausstrahlungøvermögen zugeschrieben werden kōnnte, dagegen wurde gefunden, dass Feuchtigkeit die normale Ausstrahlung vergrössert, während Trocknen dieselbo verminderte. Eine Untersuchung der dritten Frage gestattete den Schluss, dass die chemische Natur der Ausstrahlung die Eigenschaft der chemischen Unveränderlichkeit (chemical inertness) zeigt, wolche charakteristisch für die Elemente der Argongruppe ist. Es warde ferner gefanden, dass Lōsungen, welche von der Thorerde durch Ausfâllung mittels Ammoniak befreit wurden, immer noch Ausstrahlungsfähigkeit und Radioactivităt besitzen.

F. D. Chattaway uod J. M. Wadmore berichten über die Constitution der Hydrocyan-, Cyan- und Cyanursäuren. Ein Studium der Cyanbalogeno zeigt, dass dieselben die typischen und charakteristischen Eigenschaften von Verbindungen haben, in welchen das Halogen an den Stickstoff gebunden ist, und dass dieselben als
Iminoderivate angesehen werden müssen. Die Leichtigkeit, mit welcher die Cyanhalogene aus den Cyaniden gebildet werden bōnnen und umgekehrt, macht für die Cyanide die Iminoconstitution im höchsten Grade wahrscheinlich. Die Beziehung der Cyanide und Cyanbalogene zu der Cyansăure und ihren Salzen macht für diese ebenfalls die Isoconstitution wabrscheinlich. Die Cyanursāure bildet ein Halogenderivat $\mathrm{C}_{3} \mathrm{O}_{3} \mathrm{~N}_{3} \mathrm{Cl}_{3}$, dessen Verbalten zeigt, dass das Cl-Atom in Uun Stickstoff gebunden ist. Da Cyanurate leicht und vollstāndig in dieses Trichloriminoderivat übergefūhrt werden können, so muss fūr die Cyanursäure und ihre Derivate eine Iminoconstitution angenommen werden. Ein Studium der Cyanurchlorido und - Bromide zeigte, dass in diesen das Halogen an den $\mathrm{C}$ gebunden ist.

J. T. Hewitt und T. S. Moore lesen über eine Modification des Zeisel'schen Apparates zur Bestimmung von Methoxylgruppen. Sie ersetzen den mit $40^{\circ}$ warmem Wasser versehenen Kühler durch eine Fractionirsäule, welche so wirksam ist, dass die Potascheabsorptionskugeln weggelassen werden kōnnen und dio Zeit für die Ausführung des Versuchs betrăcbtlich vermindert wird.

W. C. Ball hat eine neue Farbenreaction a uf Hydroxylamin entdeckt. Mit gelbem Ammoniumsulfid, Ammoniak und Alkohol entsteht eine intensiv purpurrothe Färbung von charakteristischem Absorptionsspectrum. $1 \mathrm{~Tb}$. Hydroxylamin in $500000 \mathrm{~Tb}$. Wasser kann damit noch nachgewiesen werden.

A. W. C. Moenzios liest über die Empfindlichkoit oines Thermoregulators. Mit einem Regulator von 3,1 mm Bohrung konnte eine Temperatur ron $18^{\circ}$ innerhalb $0,008^{\circ}$ für 24 Tage aufrecht erbalten werden; wurde die Bohrung auf $1,9 \mathrm{~mm}$ reducirt, wobei jedoch das Gas filtrirt und getrocknet werden musste, so verringerte sich die Schwankung auf $0,0025^{\circ}$.

Die folgenden Vortrāge wurden als gelesen 